\title{
Renewable Energy Support Policy based on Contracts for Difference and Bilateral Negotiation
}

\author{
Hugo Algarvio ${ }^{1,2}$, Fernando Lopes ${ }^{1}$, João Santana ${ }^{2,3, \star}$ \\ 1 LNEG-National Laboratory of Energy and Geology, \\ Est. Paço do Lumiar 22, Lisboa, Portugal. fernando.lopes@lneg.pt \\ 2 Instituto Superior Técnico, Avenida Rovisco Pais 1, Lisboa, Portugal \\ \{hugo.algarvio, jsantana\}@tecnico.ulisboa.pt \\ 3 INESC-ID, Rua Alves Redol 9, Lisboa, Portugal
}

\begin{abstract}
The European Union has been one of the major drivers of the development of renewable energy. The energy policies of most European countries have involved subsidized tariffs, such as the feed-in tariff in Portugal, the regulated tariff and the market price plus premium in Spain, and the Renewables Obligation in UK, that came into effect in 2002. Recently, UK has made some reforms and started to consider contracts for difference (CfDs) as a key element of the energy policy. This paper presents a support policy based on CfDs and bilateral negotiation. The first phase consists in a CfD auction and the second phase involves a bilateral negotiation between a Government and each of the selected investors. The paper also presents a case-study to analyze the potential benefits of the support policy. It was performed with the help of the MATREM system. The preliminary results indicate some advantages for the Government (and, in some cases, for the investors as well).
\end{abstract}

Keywords: Energy markets, renewable energy, contracts for difference, feed-in tariffs, price risk, MATREM system.

\section{Introduction}

The energy industry has evolved into a liberalized industry in which market forces drive the price of electricity. Three markets models have been distinguished $[1,2]$ : pools, bilateral contracts and hybrid models. A pool is defined as a centralized marketplace that clears the market for buyers and sellers. Market entities submit offers for the quantities of power that they are willing to trade. These offers are handled by a market operator, whose function is to coordinate and manage the transactions between market entities. Bilateral contracts are agreement between two parties to trade electrical energy. The hybrid model combines several features of the previous two models.

\footnotetext{
* This work was supported by "Fundação para a Ciência e Tecnologia" with reference UID/CEC/50021/2019.
} 
There are various types of contractual arrangements that fall under the broad heading of bilateral contracts, notably contracts for difference (CfDs). Such contracts are bilateral agreements to provide a specific amount of energy for a fixed price, referred to as the strike price. Also, CfDs are typically indexed to a reference price, which is often the centralized market-clearing price. In some cases, CfDs can be one way contracts, when the difference payments are made by one of the parties only $[3,4]$.

The evolution of renewable energy has increased substantially over the past decade. Europe has been at the forefront of the renewable energy policy design and deployment $[5,6]$. In particular, Portugal has benefited from a feed-in tariff [7]. In Spain, two different types of retribution have been considered: the regulated or feed-in tariff and the market price plus premium. In UK, there have been two main policy instruments: the Non-Fossil Fuel Order, a centralized bidding system that ran from 1990 to 1998, and the Renewables Obligation, that came into effect in 2002. Recently, UK has made some reforms to meet the challenges of the electricity sector. Contracts for difference are a key element of such reforms - they are essentially private law contracts between low carbon electricity generators and a Government-owned company. CfDs provide long-term price stabilisation to low carbon plants, allowing investment to come forward at a lower cost of capital and therefore at a lower cost to consumers $[8,9]$.

Renewable generation is characterized by high fixed capital costs but nearzero variable production costs, and a great dependence on weather conditions. These characteristics may significantly influence the behavior and outcomes of energy markets. In particular, high levels of renewable generation may reduce market prices due to their low-bid costs, and increase price volatility because of their increased variability and uncertainty [11].

Against this background, this paper presents a study to investigate the potential benefits of both contracts for difference and bilateral negotiation as a basis of a renewable energy support policy. It considers a particular Government (e.g., the Portugal or Spain Governments) that makes a public announcement of new investment in wind energy involving two phases. The first phase consists in a CfD auction, like the UK CfD auctions $[8,10]$, when the investors make their offers. This phase is simulated by considering a contract net protocol. The Government selects all the offers that provide a specific level of benefit and comply with the requirements. In the second phase, there is a bilateral negotiation between the Government and each of the selected investors, where the parties negotiate a mutually acceptable value for the strike price. Negotiation involves an iterative exchange of proposals and counter-proposals. The study is conducted with the help of an agent-based tool, called MATREM [12,13].

The remainder of the paper is structured as follows. Section 2 presents an overview of the markets supported by the MATREM system, placing emphasis on the bilateral marketplace for negotiating long-term contracts (notably contracts for difference). Section 3 discusses some aspects of the formalization of bilateral negotiation involving CfDs. Section 4 presents the case-study and discusses the experimental results. Finally, concluding remarks are presented in section 5. 


\section{MATREM: Overview of the Bilateral Marketplace}

MATREM (for Multi-Agent TRading in Electricity Markets) is an energy management tool for simulating liberalized electricity markets. The tool supports a day-ahead market, a shorter-term market (an intra-day market), a balancing market, a futures market, and a bilateral marketplace. Market entities are modeled as software agents equipped with models of individual and social behaviour, enabling them to be pro-active (i.e., capable of exhibiting goal-directed behaviour) and social (i.e., able to communicate and negotiate with other agents in order to complete their design objectives). Currently, the tool supports generating companies, retailers, aggregators, coalitions of consumers, traditional consumers, market operators and system operators. A detailed description of the tool is presented in [12]. A classification of the tool according to various dimensions associated with both energy markets and software agents can be found in [13]. This section gives an overview of the markets supported by the tool, particularly the bilateral marketplace.

The day-ahead market is a central market where generation and demand can be traded on an hourly basis [14]. The intra-day market is a short-term market and involves several auction sessions. Both markets are based on the marginal pricing theory. The balancing market is a market for primary, secondary and tertiary reserve. For the particular case of tertiary reserve, two computer simulations are performed, one for determining the price for up-regulation, and another for computing the price for down-regulation.

The futures market is an organized market for standardized financial and physical contracts. Such contracts may span from days to years and typically hedge against the price risk inherent to day-ahead and intra-day markets. Players enter orders involving either bids to sell or buy energy in an electronic trading platform that supports anonymous operation. The platform automatically and continuously matches the bids likely to interfere with each other.

The bilateral marketplace allows market participants to negotiate all the details of two different types of tailored (or customized) long-term bilateral contracts, namely forward contracts and contracts for difference (see, e.g., [15]). Forward bilateral contracts are agreements between two agents to exchange a specific energy quantity at a certain future time for a particular price. Contracts for difference are agreements in which each agent ensures the other against discrepancies between the contract price (or strike price) and the market-clearing price. The terms and conditions of both types of contracts are flexible and can be negotiated privately to meet the objectives of two parties. To this end, market agents are equipped with a model that handles two-party and multi-issue negotiation $[16,17]$. Negotiation proceeds by an iterative exchange of offers and counter-offers. An offer is a set of issue-value pairs, such as "energy price $=50$ $€ / \mathrm{MWh}$ ", "contract duration $=6$ months", and so on. A counter-offer is an offer made in response to a previous offer. The bilateral marketplace and the associated negotiation of long-term contracts represents a novel and powerful tool. Accordingly, some details about the negotiation process follow. 
Let $A=\left\{a_{1}, a_{2}\right\}$ be the set of software agents participating in negotiation. The agent interact according to the rules of an alternating offers protocol [18]. This means that one offer (or proposal) is submitted per time period, with an agent $a_{i} \in A$ offering in odd periods $\{1,3, \ldots\}$, and the other agent $a_{j} \in A$ offering in even periods $\{2,4, \ldots\}$. The negotiation process starts with $a_{i}$ submitting a proposal $p_{i \rightarrow j}^{1}$ to $a_{j}$ at time period $t=1$. The agent $a_{j}$ receives $p_{i \rightarrow j}^{1}$ and can either accept it, reject it and opt out of the negotiation, or reject it and continue bargaining. In the first two cases, negotiation comes to an end. Specifically, if the proposal is accepted, negotiation ends successfully. Conversely, if the proposal is rejected and $a_{j}$ decides to opt out, negotiation terminates with no agreement. In the last case, negotiation proceeds to the next time period $t=2$, in which $a_{j}$ makes a counter-proposal $p_{j \rightarrow i}^{2}$. The agent $a_{i}$ receives the counter-proposal $p_{j \rightarrow i}^{2}$ and the tasks just described are repeated, i.e., $a_{i}$ may either accept the counter-proposal, reject it and opt out of the negotiation, or reject it and continue bargaining. Negotiation may end with either agreement or no agreement.

Negotiation strategies are computationally tractable functions that define the negotiation tactics to be used during the course of negotiation. Concession tactics are functions that generate new values for each issue at stake throughout negotiation. Let $X$ designate an issue and denote its value at time $t$ by $x$. Formally, a concession tactic for $X$ is a function with the following general form:

$$
Y(x)=x+(-1)^{m} C_{f}(x-\lim )
$$

where $m=0$ if an agent $a_{i}$ wants to minimize $X$ or $m=1$ if $a_{i}$ wants to maximize $X, C_{f} \in[0,1]$ is the concession factor, and $\lim$ is the limit for $X$ (i.e., the point where $a_{i}$ decides to stop the negotiation rather than to continue, because any agreement beyond this point is not minimally acceptable). The concession factor $C_{f}$ can be a positive constant independent of any objective criteria. Also, $C_{f}$ can be modelled as a function of a single criterion. Useful criterion include the total concession made on each issue throughout negotiation as well as the amount or quantity of energy for a specific trading period [19].

\section{Bilateral Negotiation and Contracts for Difference}

As noted, contracts for difference are agreements in which the purchaser pays the seller the difference between the contract price (or strike price) and some reference price, usually the market-clearing price. Concretely, CfDs are settled as follows [1]:

- if the strike price is higher than the market-clearing price, the buyer pays the seller the difference between these two prices times the energy quantity agreed;

- conversely, if the strike price is lower than the clearing price, the seller pays the buyer the difference between these two prices times the quantity of energy agreed. 
In this section, we consider that CfDs may specify the provision of different quantities of energy for different periods of time, at different prices (see also [19]). Thus, we consider that the set of negotiating issues includes $n$ strike prices and $n$ energy quantities. Let $P_{k}$ be a strike price and $Q_{k}$ an energy quantity (for $k=1, \ldots, n)$. Let $p_{k}$ denote the value of $P_{k}$ for quantity $q_{k}$ of $Q_{k}$. Also, let $r p_{k}$ be the value of a reference price $R P_{k}$ associated with a specific period of a day. The financial compensation associated with CfDs can now be formalized. Specifically, when the strike prices are smaller than the reference prices, the amount to pay is as follows:

$$
C_{s}=\sum_{k=1}^{n}\left(r p_{k}-p_{k}\right) \times q_{k}
$$

And the amount to pay by buyers to sellers is as follows:

$$
C_{b}=\sum_{k=1}^{n}\left(p_{k}-r p_{k}\right) \times q_{k}
$$

\section{Case-Study}

This section analyzes the potential benefits of a renewable energy support policy involving two phases. The agents are a Government of a particular country (e.g., Portugal or Spain) and various investors (or renewable energy producers). The first phase consists in a CfD auction, like the UK CfD auctions, where investors can make offers, and the Government select the best ones according to pre-defined requirements. The second phase consists in a private bilateral negotiation between the Government and each of the selected investors, to obtain a mutually acceptable value for the strike price. Since each selected investor knows the first proposal of the other investors, there is the possibility to make a more competitive offer in this phase. Accordingly, this support policy could be advantageous for the Government (and, in some cases, for the investors as well).

First Phase: CfD Auction. The public announcement involves the investment in wind energy in a maximum of $100 \mathrm{MW}$ of installed capacity (per investor). The investors can propose projects involving less than $100 \mathrm{MW}$, but only in group with investors in a similar situation. Joint projects involving more than $100 \mathrm{MW}$ of installed capacity can be accepted, in case they are advantageous for the Government. All investors should submit the following: strike price (SP), average expected power factor (PF) of the project, and (iii) installed capacity (IC). The power factor consists in the average number of hours that wind farms will be operating at the installed capacity/nominal power.

The points $(\mathrm{P})$ attributed to each proposal are computed by considering the relation between the strike price and the average expected power factor, as well as the installed capacity (see Equation 4 ). The power factor and the strike price have weights $k_{1}$ and $k_{2}$, respectively (such that $k_{1}+k_{2}=1$ ). Since SP is often the most important factor, in this work we consider $k_{1}=0.3$ and $k_{2}=0.7$. 
Table 1. CfD auction results: projects accepted for the next phase.

\begin{tabular}{ccccc}
\hline $\begin{array}{c}\text { Project } \\
\text { Id }\end{array}$ & $\begin{array}{c}\text { Strike price } \\
(€ / \mathrm{MWh})\end{array}$ & $\begin{array}{c}\text { Power factor } \\
(\%)\end{array}$ & $\begin{array}{c}\text { Installed capacity } \\
(\mathrm{MW})\end{array}$ & Points \\
\hline 9 & 74.20 & 31.90 & 100.00 & 18.84 \\
2 & 73.85 & 31.90 & 30.00 & 18.14 \\
3 & 75.80 & 31.30 & 80.00 & 18.05 \\
1 & 73.90 & 29.50 & 100.00 & 17.96 \\
11 & 75.70 & 30.20 & 100.00 & 17.95 \\
\hline
\end{tabular}

$$
P=100 \times\left(\frac{k_{1} P F}{k_{2} S P} \times\left(1.05-\frac{1-\frac{I C}{100}}{10}\right)\right)
$$

The CfD auction involved a total of 15 proposals, but only 5 were accepted for the next phase. Specifically, projects 1, 9 and 11 were accepted as individual projects, while projects 2 and 3 were accepted as a joint project. This means that investors of projects 2 and 3 make a strategic alliance to be awarded a CfD (although their companies remain separated). Table 1 shows the CfD allocation auction round outcome, with the 5 projects delivering $410 \mathrm{MW}$ of renewable energy. Several potential projects for the next delivery years did not get awarded a CfD, which may to some extent raise questions about the viability of the CfD regime for small and medium enterprises. Probably, a strike price of around 80 $€$ is acceptable, but lower than this could be not workable. Furthermore, the importance of interest rates in relation to the strike price should not be ignored in future work, given that the project time line will likely extend into periods of possible interest rate change which could impact on the viability of any strike prices.

Second Phase: Private Bilateral Negotiation. After announcing the results of the CfD auction, the Government starts private bilateral negotiations with the investors. For projects 1, 9 and 11, negotiation involves the Government and each of the corresponding investors. Projects 2 and 3 are a joint project, meaning that negotiation involves the Government and the agent representing the joint project. A detailed description of the negotiation process between the Government and the investor responsible for project 9 follows.

Negotiation proceeds through two main phases: a beginning or initiation phase (pre-negotiation) and a middle or problem-solving phase (actual negotiation). Pre-negotiation involves the creation of a well-conceived plan specifying the issues at stake, the limits and targets, the attitude toward risk and an appropriate strategy. In this work, the negotiating agenda involves mainly the strike price. The limit or resistance point is the point where each party decides to stop the negotiation rather than to continue. The target point is the point where each negotiator realistically expects to achieve an agreement. We consider that the Government adopts a risk-seeking attitude. Thus, assuming the existence of a 
number of interested investors, the Government can adopt an aggressive position throughout negotiation in searching for a good deal. The investor adopts a risk-averse attitude, acting carefully to achieve a deal and award a CfD. Both parties adopt concession strategies, meaning that they are willing to partially reduce their demands to accommodate the opponent.

Actual negotiation involves an iterative exchange of offers and counter-offers. The investor makes an opening offer involving the strike price shown in Table 1 $(74.20 € / \mathrm{MWh})$. The Government may be pleased with such an offer, but might still believe that there is room for a few concessions. Accordingly, the Government responds with an offer involving a strike price lower than the received price, lets say $72.50 € / \mathrm{MWh}$. After these two offers, the parties may argue for what they want, but at a certain point they recognize the importance of moving toward agreement. Thus, and despite the fact of being reluctant to make any concession, the investor slightly reduces the strike price. The Government decides to respond in kind and mirror the concession of the investor. And the agents enter into a negotiation dance, exchanging proposals and counter-proposals, until they

reach the final price of $73.37 € / \mathrm{MWh}$. This price represents a good deal for the Government and an acceptable deal for the investor, who will be awarded with a contract for difference for the next 15 years.

\section{Conclusion}

This article has presented an energy support policy based on contracts for difference and bilateral negotiation. The support policy involves two phases. The first consists in a CfD auction, like the UK auctions, and the second involves a private bilateral negotiation between each of the selected investors and a particular Government.

Preliminary results from a case-study indicate some advantageous for the Government (and, in some cases, for the investors as well). In the future, we intend to perform a number experiments, using controlled experimentation as the experimental method, to evaluate the effectiveness of the support policy in a number of different situations.

\section{References}

1. Kirschen, D., Strbac, G.: Fundamentals of Power System Economics, John Wiley \& Sons, Chichester, 2018.

2. Lopes, F., Coelho, H.: Electricity Markets with Increasing Levels of Renewable Generation: Structure, Operation, Agent-based Simulation and Emerging Designs, Springer, Cham, 2018. https://doi.org/10.1007/978-3-319-74263-2

3. Stoft, S.: Power Systyem Economis: Designing Markets for Electricity, IEEE Press and Wiley Interscience (2002)

4. Lopes, F.: Electricity Markets and Intelligent Agents Part I: Market Architecture and Structure. In: Electricity Markets with Increasing Levels of Renewable Generation: Structure, Operation, Agent-based Simulation and Emerging Designs, pp. 23-48, Springer, Cham, 2018. 
5. European Union: Directive 2009/28/EC of the European Parliament and of the Council on the promotion of the use of energy from renewable sources and amending and subsequently repealing Directives 2001/77/EC and 2003/30/EC (23 April 2009)

Available at: http://eur-lex.europa.eu/legal-content/EN/TXT/?uri=CELEX:32009L0028. (access date: February 2020)

6. European Union: The Promotion of the use of Energy from Renewable Sources, Directive (EU) 2018/2001, 11 December 2018.

Available at: http://data.europa.eu/eli/dir/2018/2001/oj. (access date: February 2020)

7. Lopes, F., Sá, J., Santana, J.: Renewable Generation, Support Policies and the Merit Order Effect: A Comprehensive Overview and the Case of Wind Power in Portugal, In: Lopes F., Coelho H. (eds), Electricity Markets with Increasing Levels of Renewable Generation: Structure, Operation, Agent-based Simulation, and Emerging Designs, Studies in Systems, Decision and Control, vol 144., pp. 227263, Springer, Cham, Switzerland, 2018.

8. Department for Energy and Climate Change: Implementing Electricity Market Reform (June 2014)

Availabel at: https://www.gov.uk/government/publications/implementingelectricity-market-reform-emr. (access date: February 2020)

9. Grubb, M., Newbery, D.: UK Electricity Market Reform and the Energy Transition: Emerging Lessons, Working Paper Series CEEPR WP 2018-004, Massachusetts Institute of Technology, February 2018.

10. Eversheds: First Contract for Difference (CfD) auction results announced, Eversheds Sutherland International, Available at: https://www.evershedssutherland.com/global/en/what/articles/index.page?ArticleID=en/Energy/firstcfd-auction-results-announced-150226 (access date: February, 2020)

11. Ela, E., Milligan, M., Bloom, A., Cochran, J., Botterud, A., Townsend, A., Levin, T.: Overview of Wholesale Electricity Markets, In: Electricity Markets with Increasing Levels of Renewable Generation: Structure, Operation, Agent-Based Simulation and Emerging Designs, Springer International Publishing, Cham, Switzerland, pp. 3-21, 2018.

12. Lopes, F.: MATREM: An Agent-based Simulation Tool for Electricity Markets., In: Electricity Markets with Increasing Levels of Renewable Generation: Structure, Operation, Agent-Based Simulation and Emerging Designs, Springer, Cham, Switzerland, pp. 189-225, 2018.

13. Lopes, F., Coelho, H.: Electricity Markets and Intelligent Agents. Part II: Agent Architectures and Capabilities, In: Electricity Markets with Increasing Levels of Renewable Generation: Structure, Operation, Agent-Based Simulation and Emerging Designs, Springer, Cham, Switzerland, pp. 49-77, 2018;

14. Algarvio, H., Couto, A., Lopes, F., Estanqueiro, A., Santana, J.: Multi-Agent Energy Markets with High Levels of Renewable Generation: A Case-Study on Forecast Uncertainty and Market Closing Time, In: S. Omatu et al. (eds.), 13th International Conference on Distributed Computing and Artificial Intelligence, pp. 339-347. Springer International Publishing, 2016.

15. Sousa, F. Lopes, F., Santana, J.: Contracts for Difference and Risk Management in Multi-agent Energy Markets, In: Advances in Practical Applications of Agents, Multi-Agent Systems, and Sustainability: The PAAMS Collection (PAAMS 2015), Springer International Publishing, pp. 339-347, 2015. 
16. Lopes, F., Mamede, N., Novais, A. Q., Coelho, H.: A Negotiation Model for Autonomous Computational Agents: Formal Description and Empirical Evaluation, Journal of Intelligent \& Fuzzy Systems 12, pp. 195-212, 2002.

17. Lopes, F., Mamede, N., Novais, A. Q., Coelho, H.: Negotiation in a Multi-Agent Supply Chain System, In: 3rd Int. Workshop of the IFIP WG 5.7 Special Interest Group on "Advanced Techniques in Production Planning \& Control", pp. 153-168, Firenze University Press, 2002.

18. Osborne, M., Rubinstein, A.: Bargaining and Markets. Academic Press, Inc., New York (1990)

19. Lopes, F., Algarvio, H., Santana, J.: Agent-based Simulation of Electricity Markets: Risk Management and Contracts for Difference. In: Agent-based Modelling of Sustainable Behaviors, pp. 207-225, Springer, Cham (2017) 